\section{Respuestas}

Año 14

No. 2

Diciembre 2009

ISSN 0122-820X

\title{
Planisoft: herramienta web para realizar estimaciones en los proyectos de software
}

Judith del Pilar Rodríguez, Tenjo ${ }^{1} \mid$ Willinton Cruz Paez²

Recibido:

1 de julio de 2009

Aceptado:

2 de diciembre de 2009

\section{Resumen}

En este artículo se presenta la herramienta para la gestión de proyectos de software, Planisoft; herramienta desarrollada con el objetivo de permitir llevar un control para la gestión de proyectos, abarcando las etapas que contiene este, como lo son formulación, ejecución y finalización; el desarrollo del proyecto siguió una metodología iterativa e incremental orientada a objetos guiada por el proceso unificado, empleando el lenguaje UML como herramienta de modelado [1].

Palabras Clave: Gestión de proyectos, Planificación y Control, Actividades de desarrollo, Ajax, Ingeniería de Software.

\section{Abstract}

This article presents the tool for project management software, Planisoft; tool developed with the aim of allowing monitor for project management, covering the stages to contain it, such as design, implementation and completion, the development of the project followed a methodology iterative and incremental object-oriented guided by the unified process and methodology hypermedia object-oriented OOHDM, using language like UML modeling tool, and bearing in mind the stereotypes that poses Jim Conall for the user experience in his book [1].
${ }^{1}$ MSC Profesora TC Asociado Universidad Francisco de Paula Santander, Directora del Grupo de Investigación y Desarrollo de Software “GIDIS”.jprodrig@bari.ufps.edu. co,pilinrt@gmail.com

${ }^{2}$ Estudiante Ingeniería de Sistemas de la Universidad Francisco de Paula Santander, investigador de Grupo de Investigación y Desarrollo de Software "GIDIS".willintoncp@ufps.edu.co 


\section{Introducción}

$\mathrm{L}$ a Gestión de Proyectos tiene como finalidad principal planear, organizar, proveer, monitorizar, controlar las actividades y los recursos humanos y materiales que intervienen en el desarrollo de un sistema de información (IEE Std-1058.1-1987)

La planificación consiste en determinar qué se debe hacer cómo debe hacerse, quién es el responsable de que se haga y por qué.

Para cumplir lo anterior se creó este proyecto que pretende desarrollar una aplicación Web con las funciones de gestión de proyectos, permitiendo organizar la información en la ejecución de un proyecto de software desde la organización inicial hasta la fase de culminación del mismo, basados en los procesos de ingeniería del software.

Planisoft es una herramienta de software para la gestión de proyectos de software. Esta aplicación permite organizar la información acerca de la asignación de tiempos a las tareas, los costos asociados y los recursos, tanto de trabajo como materiales del proyecto, para que se puedan respetar los plazos sin exceder el presupuesto y conseguir así los objetivos planteados $[2,4,5]$.

Para ello se toma OOHDM como metodología usada, que propone el desarrollo de aplicaciones hipermedia mediante un proceso de 4 etapas: diseño conceptual, diseño navegacional, diseño de interfaces abstractas, e implementación; proporcionando un conjunto de nuevas vistas que extienden el UML para modelar únicamente la interfase de aplicaciones Web., junto con los estereotipos planteados por Conallen [1, 14].

Planisoft plantea una arquitectura de cinco capas y utiliza para su desarrollo software libre, y librerías importantes como lo son Jquery [9], Itext [10], FusionChart [11].

\section{Alcance de Planisoft}

Diciembre 2009 ISSN 0122-820X
2.1 Planificación a través de Actividades: ofrece una hoja de datos básica para la gestión de la información necesaria para llevar a cabo una actividad en el desarrollo de un proyecto de software, almacena la información asociada a esta, tal como: nombre, duración, tipo de duración, trabajo, tipo de trabajo, responsable de la actividad y una descripción relacionada con la actividad [3].

Las actividades son calendarizadas con una fecha de fin y una duración para permitir realizar estimaciones de la fecha de finalización del proyecto. Para que una actividad se ejecute es necesario que alguien la realice, por esto una actividad tiene un responsable, así como unos recursos asignados para que se pueda llevar a cabo, además las actividades son relacionadas unas con otras para poder establecer el diagrama que visualice la finalización del proyecto $[13,16]$.

Planisoft trabaja con una calendario laboral predefinido utilizado para el cálculo de la duración de una actividad, los días laborales establecidos son lunes, martes, miércoles, jueves, viernes, y sábado. Sin embargo se pueden gestionar días festivos para ser tenidos en cuenta, debido a que la aplicación realiza el cálculo de la fecha final de una actividad a partir de una fecha inicial ingresada y de una duración de la misma en unidades días[5] .

Administración de Recursos: permite clasificar los recursos en dos tipos: los recursos físicos y los recursos humanos; los físicos son todos aquellos materiales necesarios para el desarrollo del proyecto, mientras que los humanos hace referencia al personal requerido para el desarrollo del mismo. Además permite la asignación de estos recursos a las tareas así como los costos que estos generan al proyecto.

Los recursos son calendarizados para llevar un control de su uso, a estos son necesarios 
la asignación de un costo que es registrado, a las vez se permite la relación de recurso con tarea.

Administración de Tareas: las actividades en Planisoft están compuestas de tareas, estas son gestionadas a través de una hoja de datos básica que permite su relación con una actividad, así como su asignación de breve descripción para su fácil identificación.

Riesgos: losriesgospuedenseradministrados con el esquema básico, donde son listados, clasificados, asignando una probabilidad de ocurrencia y además almacena una serie de pasos a seguir en caso de que uno de los riesgos se llegase a presentar.

Las características que se almacena con relación a un riesgo es: nombre del riesgo, categoría del cliente, probabilidad de ocurrencia e impacto que causa dentro del desarrollo o realización del proyecto. El impacto y la categoría se pueden personalizar.

Actividades de Calidad: ofrece un espacio de trabajo para el manejo de tareas de calidad que se deben tener en cuenta en la realización de un proyecto de software, la cual es manipulada de forma similar a las tareas, solo que están son relacionadas a una tarea u actividad especifica, para revisar la calidad en el desarrollo del proyecto de software.

Costos: Planisoft ofrece un espacio de trabajo para gestionar los costos, ya que en el desarrollo de una actividad es necesario incurrir en unos costos. Planisoft cuenta con dos tipos de costos, los costos fijos son aquellos necesarios para el desarrollo de una cosa dentro del plan de trabajo, y los costos variables aquellos que dependen del tiempo o se encuentran en dependencia de este. Esta característica se encuentra inmersa en las actividades y tareas.

Pruebas: se ofrece un espacio de trabajo para gestionar las pruebas que se van a realizar durante la ejecución del proyecto de software, ya que son necesarias para cumplir con el objetivo del proyecto que se este ejecutando, las pruebas deben ser relacionadas ya sea con una tarea o actividad en la cual se vaya a practicar dicha prueba.

Diagramas: el seguimiento del plan se puede gestionar en base a varios diagramas de Gantt y de Pert; el diagrama principal es el de Gantt, permitiéndole a la Planisoft visualizar a medida que se ingresa la información relaciona con las tareas. El diagrama de Pert es un diagrama de red que proporciona la información básica de cada tarea en forma de red, nodos y relaciones entre ellos. Otro diagrama utilizado es el de torta, este diagrama es utilizado para visualizar la información del porcentaje de tareas que se encuentren completas e incompletas. El diagrama de barras permite mostrar información de aquellos costos gestionados en Planisoft, todos los diagramas son visualizados en una ventana modal para una mejor vista.

Informes: Planisoft ofrece un listado de informes para el seguimiento del plan que se está gestionando:

- Actividad: del cual se desprenden los siguientes informes:

- Actividad detallado: muestra el plan de actividades que se ha gestionado de manera específica.

- Progreso de la Actividad: permite visualizar las actividades que han completado un 100\% su ejecución.

- Costo por actividad: detalla los costos asignados a una actividad y el tipo de costo.

- Calendario actividad: muestra las fechas festivas gestionadas para la utilización del calculo de fechas finales de una actividad en Planisoft.

Recursos: del cual se desprenden los siguientes informes:

Listado de recursos: muestra cada uno de los recursos gestionados así como sus 
características principales.

- Recursos por tarea: detalla la cantidad de recursos que han sido asignados a una tarea.

- Costos por recursos: detalla los costos asignados a un recurso y el tipo de costo.

- Calendario recurso: muestra el calendario asignado a cada uno de los recursos gestionados en Planisoft.

Tareas: del cual se desprende los siguientes informes:

- Tareas por Actividad: detalla cada una de las tareas gestionadas con sus características y su relación con una actividad.

- Costo por tarea: detalla los costos asignados a una tarea y el tipo de costo.

Informe de riesgos: muestra información referente a los riegos gestionados junto a sus características:

- Listado de pruebas: detalla información de las pruebas y su relación con una actividad o tarea.

- Listado de actividades calidad: detalla información de las actividades de calidad gestionadas en Planisoft y su relación con una actividad o tarea.

- Informe general del proyecto: este informe permite obtener información global del proyecto que se esta gestionando, el informe es presentado en un archivo en formato .pdf que recopila los datos almacenados.

Estimación: Planisoft permite realizar una estimación de costo de proyecto basada en la información gestionada en lo referente a los costos ingresados de las tareas, actividades y recursos, igualmente permite realizar una estimación de costo de software basado en las técnicas de puntos de fusión y estimación basada en líneas de código.
Usuario: ofrece un espacio para la gestión de usuarios en un proyecto determinado, así como la modificación de información del usuario que se encuentre gestionando el proyecto.

Proyecto: permite la gestión de un nuevo proyecto, la modificación de datos generales de aquel se encuentre en gestión, y la apertura de otro si existiera.

Estado del Proyecto: ofrece la posibilidad de seleccionar el estado en que se encuentre un proyecto, ya sea en formulación o en ejecución, permitiendo modificar información referente a cada estado, una vez iniciada la sesión, si no se cuenta con un proyecto registrado, se permitirá la creación de un proyecto en estado de formulación, posteriormente se podrá observar su desarrolla al estar en ejecución.

2.2 Modelo del dominio. Este Modelo agrupa la funcionalidad del Planisoft, se organiza: proyecto, quien organiza el plan a través de tareas, por esto un proyecto tendrá muchas tareas, a su vez las tareas tiene asociado costos y recursos, el proyecto puede tener muchos riesgos, actividades para la calidad, varios usuarios y visualiza la información a través de diagramas[1,6]. La figura 1 muestra el modelo del dominio de Planisoft.

Figura 1. Modelo del dominio de Planisoft.

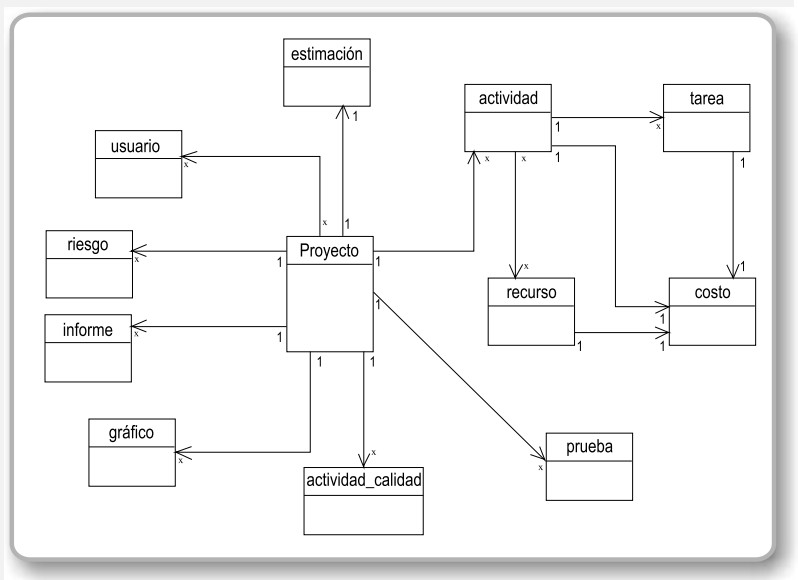

estimación Diciembre 2009 ISSN 0122-820X

(1)




\subsection{Ingeniería de requerimientos.}

El proceso de recopilar, analizar y verificar las necesidades del cliente o usuario para un sistema es llamado ingeniería de requerimientos. La meta de la ingeniería de requerimientos (IR) es entregar una especificación de requisitos de software correcta y completa [6].

Algunos de los requerimientos definidos son:

2.3.1 Requerimientos Funcionales (RF).

$\mathrm{R} 1$. Ingresar y modificar los datos generales de un proyecto, así como su respectivo almacenamiento.

R2. La administración de claves para el ingreso de las personas vinculadas al proyecto que se este desarrollando.

R3. Crear, editar, eliminar tareas, actividades, recursos, pruebas, riesgos, actividades de calidad, para el desarrollo del plan que se lleve a cabo.

R4.Listar y clasificar recursos, humanos (personal, la gente relacionada con la realización del Plan de proyecto) y físicos (hardware y componente físicos necesarios para llevar a cabo el plan) para el desarrollo del Plan.

R5. Asignar los recursos a las tareas previamente listadas.

R6. Realizar la estimación de costo del proyecto.

R8. Crear, editar, eliminar gestionar los riesgos de cada proyecto.

R9. La aplicación deberá proporcionar un calendario para la programación del tiempo de duración de tareas y actividades.

R10. ingresar y consultar los costos de las actividades, recursos y del proyecto.

R11.Listar, documentar y hacer seguimiento a las actividades estimadas para la calidad del proyecto.

R12. La emisión de informes para el seguimiento del Plan.

R14. Visualizará los Diagramas de Gantt, Pert, de barras y de torta para ver los datos del proyecto.

R15. Planisoft genera las fechas finales tanto de actividades como de tareas, a partir del ingreso de una fecha inicio y unos días de duración.

R16. La aplicación permite el manejo de estados de proyectos, formulación, ejecución, culminación.

R17. La aplicación permite gestionar los días festivos para el cálculo de fechas finales de actividades y tareas.

R18. La aplicación permite el manejo de proyectos en estado de ejecución a través de un menú personalizado.

R19. Planisoft realiza el cálculo de complejidad de proyecto, asignando un valor bajo, medio y alto a cada una de las actividades gestionadas, la complejidad será asignada dependiendo de la mayor cantidad de actividades con una complejidad dada.

RF20. La aplicación proporciona formularios para la gestión de pruebas y actividades de calidad para un proyecto.

RF21. Planisoft permite la visualización de las propiedades de cada proyecto, así como de actividades, tareas, recursos, entre otros.

\subsubsection{Requerimientos no funcionales (RNF).}

RNF1. La aplicación es rápida, la consulta a los datos ingresados y el acceso a la información se obtienen de una forma ágil y precisa.

RNF2. La aplicación funciona con una base de datos para todos los proyectos que se cree, la cual será gestionada con el motor de base de datos de PostgreSql.

RNF3. La aplicación ofrece una ayuda, para el ingreso de información en cada interfaz de usuario.

RNF4. la aplicación necesita de la maquina virtual de Java para el desarrollo de la aplicación.

RNF5. Se necesita del servidor Web Apache Tomcat.

RNF5. Se necesita de los Drivers propios del motor de base de datos para el acceso a los datos a través de JDBC.

2.4 Escenarios del Usuario. Conforme se comenzó a materializarse la función de Planisoft se empezó a definir los actores y crear 
el conjunto de escenarios que identificaron la cadena de casos de uso a construir para Planisoft [7] ver figura 2.

Actores del sistema. Se identifica como actor principal el usuario.

Usuario: Es cualquier persona que ingresa al Planisoft con el fin de realizar una planificación dentro de la gestión de un proyecto de software.

Gestor: El gestor es el Ingeniero ó persona vinculada con la Ingeniería del Software, quien se encarga principalmente de dirigir y/o planificar el desarrollo de un proyecto de software.

Administrador: persona delegada cuya función es administrar el sistema Planisoft.

Figura 2. Diagrama de caso de uso de Planisoft

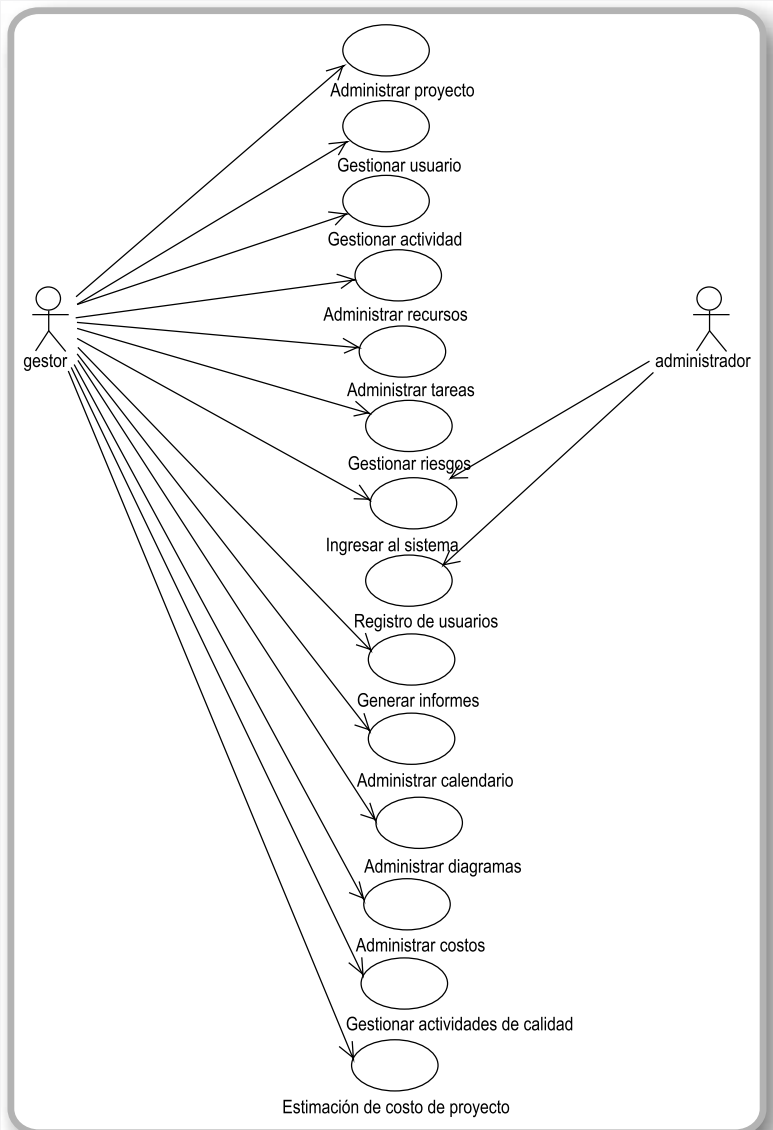

2.4.1 Diagramas de casos de uso del sistema en paquetes. La funcionabilidad que

presenta Planisoft fue resumida en paquetes, donde cada paquete almacena y organiza la información según la función a la que su nombre hace mención [7], es decir, el paquete Gestionar Usuarios, se encarga de realizar los procesos y funciones para la creación, edición y eliminación de usuarios, ver Figura 3.

Seguidamente se desarrollaron los artefactos donde se describen los paquetes del sistema y diagramas de casos de uso para cada subsistema y sus especificaciones de Planisoft.

Figura 3. Artefacto paquete principal de Planisoft

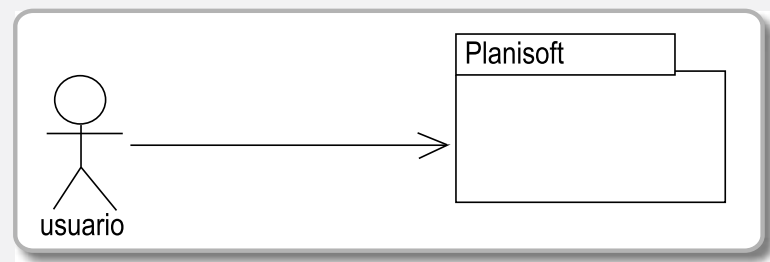

\section{Construcción del modelo de análisis}

$\mathrm{S} \longrightarrow$

Durante la etapa de análisis de Planisoft, se plantea cada uno de los requerimientos funcionales, plasmando a Planisoft, como una herramienta que abarcara el manejo de actividades y tareas, administración de recursos y de riesgo, gestión de actividades de calidad, manejo de costos por actividades y total del proyecto, y además planteando generación de informes gráficos de todos los datos almacenados.

El propósito fundamental del modelo de análisis fue resolver los requisitos definidos para Planisoft con mayor profundidad, estos fueron estructurados de modo que facilitará su comprensión, su preparación, modificación y en general, su mantenimiento. Diciembre 2009 (1) 
sus dependencias y contenidos. Las clases del análisis, sus responsabilidades, atributos, relaciones y requisitos especiales.

Los casos de uso se refinaron en término de colaboraciones dentro del modelo del análisis.

\section{Modelo del diseño}

El modelo de diseño permitió describir la realización física de los casos de uso centrándose en los requisitos funcionales y no funcionales.

Diseño navegacional. Aquí se definió la estructura de navegación a través del hiperdocumento mediante la realización de modelos navegacionales que representen diferentes vistas del esquema conceptual de la fase anterior. Se trata de reorganizar la información para adaptarla a las necesidades de los usuarios del sistema.

Diseño de la interfase abstracta, permitió especificar la estructura y el comportamiento de la Interfase del sistema hipermedia con el usuario. Este modelo es abstracto y, por tanto, independiente de la implementación final del sistema.

La interfaz abstracta utilizada en el desarrollo de Planisoft simplifica su navegación estandarizando la forma de presentación de información al usuario, manteniendo su atención en el contenido requerido. Ver figura 4 .

Figura 4. Diagrama de Interfaz Abstracta Principal Modelo Textual.

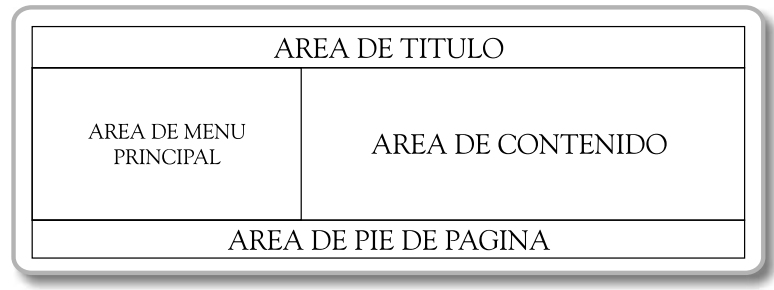

\section{Análisis de los patrones de diseño}

En Planisoft se trabajaron diversos patrones que a continuación se mencionaran, (Ajax Design Patterns de Michael Mahemoff[MAHEMOFF, 2006]) [10] .

Patrón XMLHttpRequest Call. Este patrón permitió la creación de un objeto XMLHttpRequest con el objeto de que un navegador web pueda realizar una comunicación asincrónica con el servidor utilizado, gracias a XMLHttpRequest, disponible en todos los principales browsers Javascript, el código HTTP puede hacer llamadas de nuevo a su servidor de origen y obtener de los resultados; a diferencia de la forma convencional de las presentaciones, lo que causa una página completa de refresco.

Patrón Cross-Browser Component. Crear componentes cross-browser, lo que permite a los programadores es la reutilización sin respecto de la idiosincrasia del navegador. Un componente cross-browser basado en el componente XMLHttpRequest usado en la tecnología Ajax [8], permite asegurar que la comunicación entre el navegador y el servidor se ejecute sin importar el browser utilizado, para ello se debe especificar el tipo de instanciación del objeto XMLHttpRequest que va a ser declarado para la ejecución de consultas asincrónicas [11, 12, 15].

\section{Modelo de implementación}

El modelo de implementación de Planisoft se organizo por paquetes según su función. Los cuales se presentan en las figuras 5 y 6 .

Figura 5. Modelo de implementación de usuarios

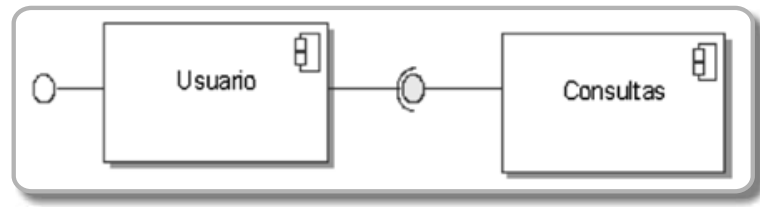


Figura 6. Modelo de implementación tarea

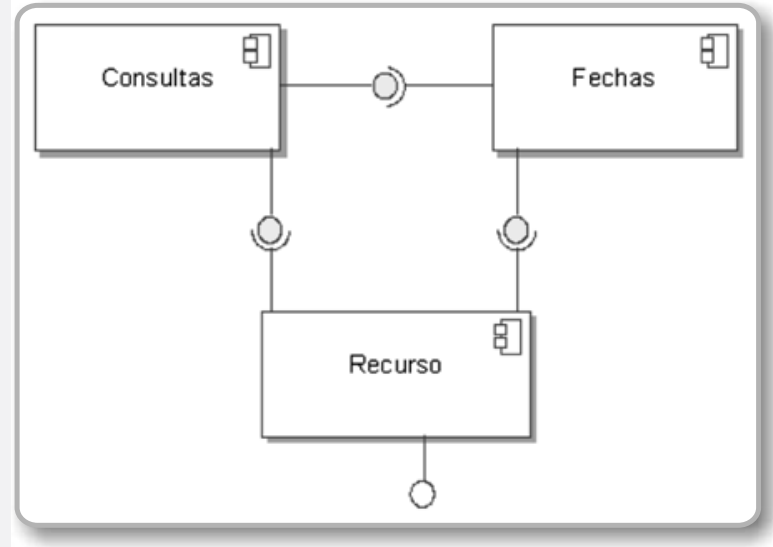

\section{Arquitectura del \\ sistema}

PlaniSoft se basa en una arquitectura de cinco (5) capas tal como se muestra en la tabla 1, permitiendo dividir el trabajo de desarrollo de una Aplicación Web en problemas pequeños que pueden delegarse a grupos especializados en cada área; además permite realizar cambios y optimizar cada capa sin modificar el resto del sistema, logrando alcanzar los dos principales objetivos de la ingeniería del software, la potabilidad y la reutilización.

Tabla 1. Arquitectura en cinco capas

\begin{tabular}{|c|}
\hline CAPA ALMACENAMIENTO \\
\hline CAPA ACCESO A DATOS \\
\hline CAPA DE SERVICIOS O LOGICA DEL NEGOCIO \\
\hline CAPA DE CONTROL \\
\hline CAPA DE VISUALIZACION
\end{tabular}

Para la implementación del software de PlaniSoft las tecnologías utilizadas en cada capa se detallan en la tabla 2.
Tabla 2. Tecnologías empleadas por cada capa

La figura 7 muestra el diagrama de despliegue de la arquitectura utilizada. La aplicación tiene como interfaz un documento HTML construido por el navegador a partir de uno o más documentos XML y uno o más documentos XSLT que son proporcionados por el sistema. La interfaz permite al usuario navegar a través de documentos XML que son vistas conceptuales de los objetos del dominio, haciendo peticiones que el navegador convierte en paquetes HTTP.

Figura 7. Arquitectura Planisoft

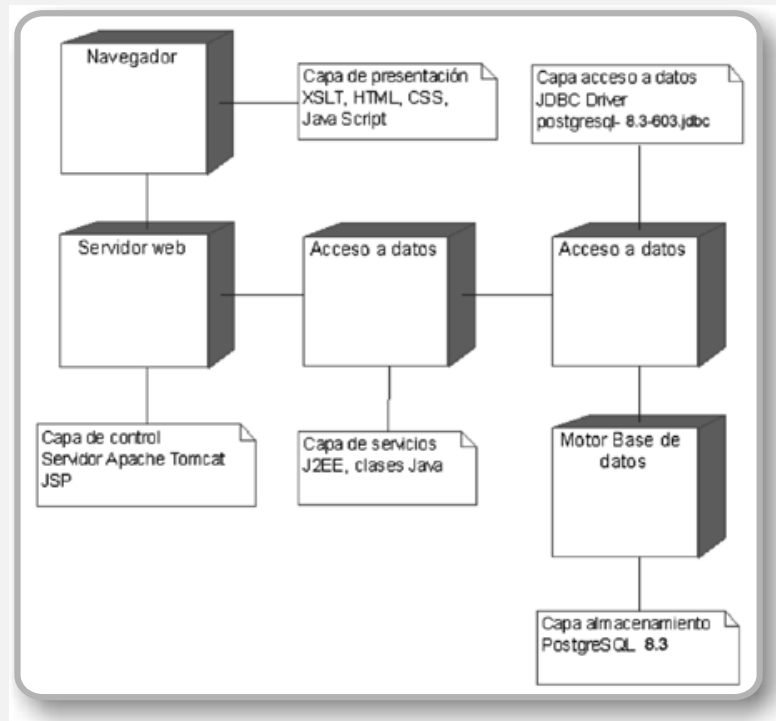


El servidor HTTP a través de los JSP toma las peticiones y retorna un documento XML y un documento XSLT al navegador. Los JSP emplean la interfaz JDBC para comunicarse con la base de datos que solucionan peticiones de usuario.

Durante la implementación se utilizaron librerías como Itext [3] que permite la creación de documentos en formato pdf, para la generación de informes, igualmente se usó Jfreechart [5] cuya utilidad fue la generación de gráficos que se implementaron en el informe de archivo pdf; Jquery [2] es una librería basada en Javascript que permite una manipulación ágil de un documento hipermedial creando efectos interesantes en su aplicación. FusionChart [4] es otra librería gráfica de carácter libre, que permite la creación de imágenes en flash a partir de datos ingresados en un documento xml.

La figura 8, muestra el interfaz principal y la figura 9, muestra la interfaz usuario gestor de proyectos.

Figura 8. Prototipo de interfaz principal.

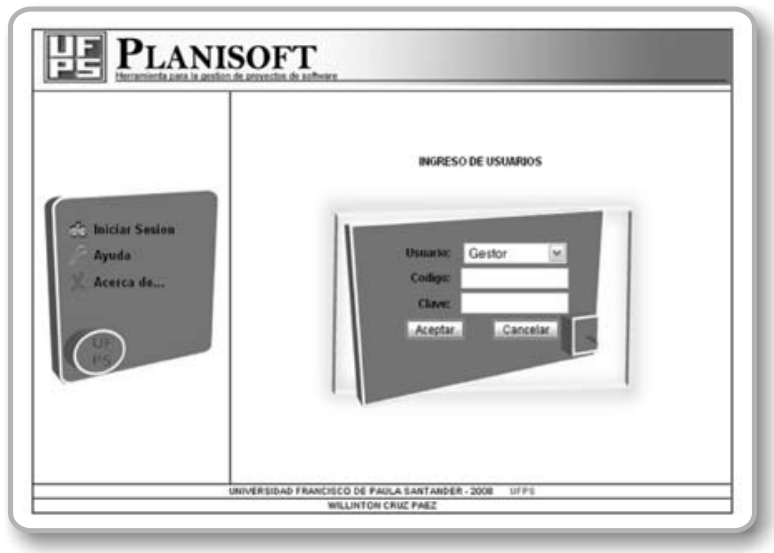

Figura 9. Prototipo de interfaz usuario gestor.

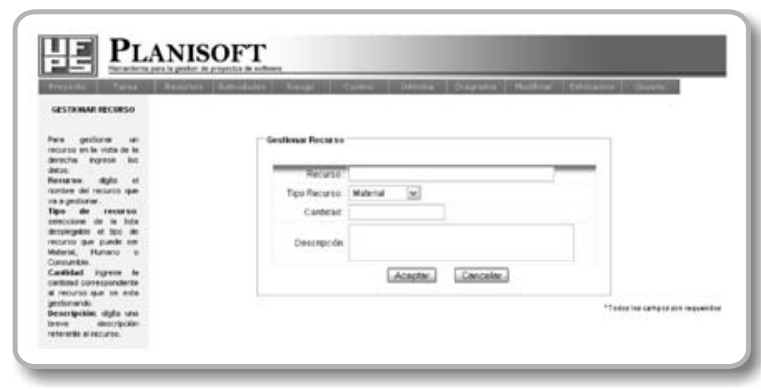

\section{Conclusiones}

La gestión de Software es una de las actividades más importantes a la hora de desarrollar proyectos de software, esta actividad involucra todo los procesos de desarrollo y control que se requieren para la formulación y ejecución de un proyecto de software.

La aplicación de esta herramientas como es PlaniSof en los cursos de ingeniería del software de la Universidad ha ayudado a los estudiantes a controlar las actividades que están desarrollando en los proyectos de software; garantizándoles la entrega del producto en el tiempo esperado y con la mejor calidad de mismo.

La totalidad de los proyectos utilizados para el desarrollo de los talleres en el segundo semestre 2008, corresponden a sistemas que informatizan la gestión administrativa de una empresa y, por sus características similares, permiten garantizar que el nivel de complejidad de los mismos sea uniforme para los diferentes equipos de estudiantes.

La propuesta presentada en este trabajo, refuerzan en el accionar de los estudiantes conla aplicación de herramientas PnaniSoft permitiéndole asistir en la gestión de proyectos de software, mejorando considerablemente la toma de decisiones. Además, permite la adquisición de conductas profesionales.

\section{Recomendaciones y agradecimientos}

Se recomienda ampliar la opción de riesgos manejada en la aplicación, permitiendo un registro más detallado y formal para la elaboración de un plan de contingencia ante cualquier riesgo dentro de la gestión de un proyecto de software.

El manejo de informes que realiza Planisoft es variado, sin embargo se recomienda desarrollar funciones que le permita al usuario crear plantillas de presentación de estos 
informes que se piensen generar, plantillas que abarquen aspectos de encabezados, cantidad de información, ubicación de esta dentro del informe o reporte.

Los autores agradecen al Grupo de Investigaciones de Desarrollo de Ingeniería del Software de la Universidad Francisco de Paula Santander, al FINU y a la Vicerrectoría Asistente y Extensión de Investigaciones de la UFPS por su apoyo en la presente investigación.

\section{Bibliografía}

[1] J, Conallen. Building Web Applications WithUml.Boston:Addison Wesley, 2003. 468p. ISBN 0-201-73038-3

[2] R, Pressman. Ingeniería del Software, Un Enfoque Practico. 6 edición. Mc Graw Hill. 2007. pp. 227-238

[3] CAPERS Jones. Estimacion de costos y administración de proyectos de software. Dando realismo a la estimación. 2da Edición. McGraw Hill 2007

[4] CORDOBA, Linsay Cheryl y DAVILA RUIZ, Diego Alexander. Análisis, Diseño E Implementación De Una Herramienta Case Para La Gestión De Proyectos De Software. San José de Cúcuta 2006. 439 p. Trabajo de Grado. Universidad Francisco de Paula Santander. Facultad de Ingeniería. Plan de Estudios de Ingeniería de Sistemas.

[5] J. Braude. Ingeniería De Software: Una Perspectiva Orientada A Objetos. Alfaomega. 2003.

[6] Jacobson, I., Booch, G., Rumbaugh, J. (2000). El proceso unificado de desarrollo de software. Addison-Wesley, Madrid.
[7] HANNA, Phil. JSP Manual de referencia, España: Mc Graw Hill, 2002. ISBN: 84-481-3264-5

[8] MAHEMOFF, Michael. Ajax Design Patterns, O'reilly, 2006. 655 p. ISBN10: 0-596-10180-5 , ISBN-13: 978-059-610180-0 .

[9] Jquery, Homepage, http://jquery.com/ [Citado el 01 de Septiembre de 2008].

[10] Itex Homepage. http://www.lowagie. com/iText/ . [Citado el 01 de Septiembre de 2008].

[11] Fusionchart, Homepage. www. fusioncharts.com/free/ . [Citado el 01 de Septiembre de 2008].

[12] Jfreechart, Homepage , www.jfree.org/ jfreechart/. [Citado el 01 de Septiembre de 2008].

[13 ] Easy Project.Net [ONLINE] http://www.easyprojects.net/ [Citado el 01 de Septiembre de 2008] ISSN 0122-820X

[14] EGUILUZ , Javier, LIBROS WEB, Introducción a Ajax [ONLINE] http://www.librosweb.es/ajax/index. html [Citado el 01 de Septiembre de 2008]

[15] FUSIONCHARTS HomePage [ONLINE] www.fusioncharts.com/free/ [Citado el 01 de Septiembre de 2008]

[16] Gannt Project Home [ONLINE] http://ganttproject.biz/ [Citado el 01 de Septiembre de 2008] 